\title{
Second Order Power Spectrum and Nonlinear Evolution at High Redshift
}

\author{
Bhuvnesh Jain and Edmund Bertschinger ${ }^{1}$ \\ Department of Physics, MIT, Cambridge, MA 02139 USA
}

\begin{abstract}
The Eulerian cosmological fluid equations are used to study the nonlinear mode coupling of density fluctuations. We evaluate the second-order power spectrum including all four-point contributions. In the weakly nonlinear regime we find that the dominant nonlinear contribution for realistic cosmological spectra is made by the coupling of long-wave modes and is well estimated by second order perturbation theory. For a linear spectrum like that of the cold dark matter model, second order effects cause a significant enhancement of the high $k$ part of the spectrum and a slight suppression at low $k$ near the peak of the spectrum. Our perturbative results agree well in the quasilinear regime with the nonlinear spectrum from high-resolution N-body simulations.

We find that due to the long-wave mode coupling, characteristic nonlinear masses grow less slowly in time (i.e., are larger at higher redshifts) than would be estimated using the linear power spectrum. For the cold dark matter model at $(1+z)=(20,10,5,2)$ the nonlinear mass is about $(180,8,2.5,1.6)$ times (respectively) larger than a linear extrapolation would indicate, if the condition rms $\delta \rho / \rho=1$ is used to define the nonlinear scale. At high redshift the Press-Schechter mass distribution significantly underestimates the abundance of high-mass objects for the cold dark matter model. Although the quantitative results depend on the definition of the nonlinear scale, these basic consequences hold for any initial spectrum whose post-recombination spectral index $n$ decreases sufficiently rapidly with increasing $k$, a feature which arises quite generally during the transition from a radiation- to matter-dominated universe.
\end{abstract}

Subject headings: cosmology: theory - large-scale structure of universe galaxies: clustering - galaxies: formation

${ }^{1}$ Also Institute for Advanced Study, Princeton, NJ 08540 


\section{Introduction}

There exists a standard paradigm for the formation of cosmic structure: gravitational instability in an expanding universe. According to this paradigm, dark matter density fluctuations $\delta(\vec{x}) \equiv \delta \rho(\vec{x}) / \bar{\rho}$ created in the early universe lay dormant until the universe became matter-dominated at a redshift $z=z_{\text {eq }} \approx 2.5 \times 10^{4} \Omega h^{2}$ (where $\Omega$ is the present density parameter for nonrelativistic matter and the present Hubble parameter is $H_{0}=100 \mathrm{~h} \mathrm{~km} \mathrm{~s}^{-1} \mathrm{Mpc}^{-1}$ ). After this time, the density fluctuations increased in amplitude as predicted by the well-known results of linear perturbation theory (e.g., Peebles 1980; Efstathiou 1990; Bertschinger 1992), until the fluctuations became nonlinear on some length scale. Bound condensations of this scale then collapsed and virialized, forming the first generation of objects (Gunn \& Gott 1972; Press \& Schechter 1974). Structure formation then proceeded hierarchically as density fluctuations became nonlinear on successively larger scales.

At early times density fluctuations were small on the length scales of present day large-scale structure. Therefore, after the universe became matter dominated fluctuations on scales much larger than the scale of collapsed objects can be studied under the approximation of a pressureless, irrotational fluid evolving under the action of Newtonian gravity. A perturbative analysis of the fluid equations in Fourier space can then be used to study the effects of mode coupling between scales that are weakly nonlinear. This is the approach we shall follow in this paper. Nonlinear analyses in real and Fourier space are somewhat complementary in that real space analyses are best suited to studying the effect of nonlinearities on the collapse and shapes of individual objects (Bertschinger \& Jain 1993), whereas Fourier space studies provide estimates of how different parts of the initial spectrum couple and influence the evolution of statistical quantities like the power spectrum. In principle of course, the two approaches are equivalent and should give the same information. For perturbative analyses in real space see, e.g., Peebles (1980), Fry (1984), Hoffman (1987), Zaroubi \& Hoffman (1993), and references therein.

Although density fluctuations of different wavelengths evolve independently in linear perturbation theory, higher order calculations provide an estimate of some nonlinear effects. Preliminary second order analyses have led to the conventional view that in models with decreasing amounts of power on larger scales long-wavelength fluctuations have no significant effect on the gravitational instability occuring on small scales. On the other hand, it is known that under some circumstances small-scale, nonlinear waves can transfer significant amounts of power to long-wavelength, linear waves. If the initial spectrum is steeper than $k^{4}$ at small $k$ (comoving wavenumber), then small-scale, nonlinear waves can transfer power to long wavelength linear waves 80 as to produce a $k^{4}$ tail in the spectrum. 
(Zel'dovich 1965; Peebles 1980, Section 28; Vishniac 1983; Shandarin \& Melott 1990).

The question of whether power can be transfered from large to small scales was examined by Juszkiewicz (1981), Vishniac (1983), Juszkiewicz, Sonoda \& Barrow (1984), and more recently by Coles (1990), Suto and Sasaki (1991) and Makino, Sasaki and Suto (1992). Their analyses involved writing down integral expressions for the second order contribution to the power spectrum, examining their limiting forms and evaluating them for some forms of the initial spectrum. Juszkiewicz et al. (1984) examined the autocorrelation function and found that the clustering length decreases due to power transfer from large to small scales for the initial spectrum $P(k) \propto k^{2}$. However, for the cold dark matter (hereafter CDM) spectrum Coles (1990) found the opposite effect, though it is not significant unless $\sigma_{8}$ is taken larger than 1. Makino et al. (1992) have analytically obtained the second order contributions for power law spectra, and estimated the contribution for the CDM spectrum by approximating it as two power laws. Bond \& Couchman (1988) have compared the second order CDM power spectrum to the Zel'dovich approximation evaluated at the same order. Some issues of mode coupling have recently been investigated through N-body simulations in 2-dimensions (see e.g., Beacom et al. 1991; Ryden \& Gramann 1991; Gramann 1992).

We have used the formalism developed in some of the perturbative studies cited above, and especially by Goroff et al. (1986), to calculate second order contributions to the power spectrum (i.e., up to fourth order in the initial density) for the standard CDM spectrum. Second order perturbation theory has a restricted regime of validity, because once the density fluctuations become sufficiently large the perturbative expansion breaks down. For this reason N-body simulations have been used more extensively to study the fully nonlinear evolution of density fluctuations. However, perturbation theory is very well suited to address some specific aspects of nonlinear evolution and to provide a better understanding of the physical processes involved. Being less costly and time-consuming than N-body simulations, it lends itself easily to the study of different models. Perturbation theory should be considered a complementary technique to $\mathrm{N}$-body simulations, for while its validity is limited, it does not suffer from the resolution limits that can affect the latter. Hence by comparing the two techniques their domains of validity can be tested and their drawbacks can be better understood. In this paper we shall make such comparisons for the CDM spectrum.

The most powerful use of perturbative calculations is in the study of weakly nonlinear evolution out to very high redshifts, spanning decades of comoving length scales in the spectrum. Since the formulation of the perturbative expansion allows for the time evolution of the spectrum to be obtained straightforwardly, we obtain the scaling in time of 
characteristic nonlinear mass scales ranging from the nonlinear scale today, about $10^{14} M_{6}$, to about $10^{5} M_{\odot}$, the smallest baryonic mass scale likely to have gone nonlinear after the universe became matter dominated. Such an analysis cannot be done by existing N-body simulations as the dynamic range required to cover the full range of scales with adequate spectral resolution exceeds that of the current state-of-the-art.

There are two principal limitations to our analytic treatment: the first arises from the general problem that the perturbative expansion breaks down when nonlinear effects become sufficiently strong. This drawback is particularly severe in our case because the regime of validity is difficult to estimate. It is reasonable to expect that second order perturbation theory ceases to be valid when the rms $\delta \rho / \rho \gtrsim 1$, but one cannot be more precise without explicitly calculating higher order contributions.

The second kind of limitation arises from the simplifying assumptions that pressure and vorticity are negligible. On small enough scales nonlinear evolution causes the intersection of particle orbits and thus generates pressure and vorticity. Through these effects virialization on small-scales can alter the growth of fluctuations on larger scales. It is plausible that the scales in the weakly nonlinear regime are large enough that this effect is not significant. This belief is supported by heuristic arguments as well as recent studies of N-body simulations (Little, Weinberg \& Park 1991; Evrard \& Crone 1992 and references therein). We conclude that the first kind of limitation, namely the neglect of higher order contributions, or worse still, the complete breakdown of the perturbative expansion, is likely to be more severe for our results. We shall address this where appropriate and accordingly attempt to draw conservative conclusions supported by our own $\mathrm{N}$-body simulations.

The formalism for the perturbative calculation is described in Section 2. We describe the numerical results for CDM in Section 3.1 and compare them to $\mathrm{N}$-body simulations in Section 3.2. The scaling of the nonlinear scale as a function of redshift is presented in Section 3.3. The distribution of nonlinear masses is examined in Section 3.4 We discuss cosmological implications of the results in Section 4.

\section{Perturbation Theory}

In this section we describe the formalism for perturbative solutions of the cosmological fluid equations in Fourier space. Our approach is similar to that of Goroff et al. (1986). The formal perturbative solutions are then used to write down the explicit form of the second order contribution to the power spectrum. 


\subsection{General Formalism}

We suppose for simplicity that the matter distribution after recombination may be approximated as a pressureless fluid with no vorticity. We further assume that peculiar velocities are nonrelativistic and that the wavelengths of interest are much smaller than the Hubble distance $\mathrm{cH}^{-1}$ so that a nonrelativistic Newtonian treatment is valid. Using comoving coordinates $\vec{x}$ and conformal time $d \tau=d t / a(t)$, where $a(t)$ is the expansion scale factor, the nonrelativistic cosmological fluid equations are

$$
\begin{gathered}
\frac{\partial \delta}{\partial \tau}+\vec{\nabla} \cdot[(1+\delta) \vec{v}]=0, \\
\frac{\partial \vec{v}}{\partial \tau}+(\vec{v} \cdot \vec{\nabla}) \vec{v}=-\frac{\dot{a}}{a} \vec{v}-\vec{\nabla} \phi, \\
\nabla^{2} \phi=4 \pi G a^{2} \bar{\rho} \delta,
\end{gathered}
$$

where $\dot{a} \equiv d a / d \tau$. Note that $\vec{v} \equiv d \vec{x} / d \tau$ is the proper peculiar velocity, which we take to be a potential field so that $\vec{v}$ is fully specified by its divergence:

$$
\theta \equiv \vec{\nabla} \cdot \vec{v}
$$

We assume an Einstein-de Sitter $(\Omega=1)$ universe, with $a \propto t^{2 / 3} \propto \tau^{2}$. We will also assume that the initial (linear) density fluctuation field is a gaussian random field.

To quantify the amplitude of fluctuations of various scales it is preferable to work with the Fourier transform of the density fluctuation field, which we define as

$$
\hat{\delta}(\vec{k}, \tau)=\int \frac{d^{3} x}{(2 \pi)^{3}} e^{-i \vec{k} \cdot \vec{x}} \delta(\vec{x}, \tau)
$$

and similarly for $\hat{\theta}(\vec{k}, \tau)$. The power spectrum (power spectral density) of $\delta(\vec{x}, \tau)$ is defined by the ensemble average two-point function,

$$
\left\langle\hat{\delta}\left(\vec{k}_{1}, \tau\right) \hat{\delta}\left(\vec{k}_{2}, \tau\right)\right\rangle=P\left(k_{1}, \tau\right) \delta_{\mathrm{D}}\left(\vec{k}_{1}+\vec{k}_{2}\right),
$$

where $\delta_{\mathfrak{D}}$ is the Dirac delta function, required for a spatially homogeneous random density field. For a homogeneous and isotropic random field the power spectrum depends only on the magnitude of the wavevector. The contribution to the variance of $\delta(\vec{x}, \tau)$ from waves in the wavevector volume element $d^{3} k$ is $P(k, \tau) d^{3} k$.

Fourier transforming equations (1) gives

$$
\frac{\partial \hat{\delta}}{\partial \tau}+\hat{\theta}=-\int d^{3} k_{1} \int d^{3} k_{2} \delta_{\mathrm{D}}\left(\vec{k}_{1}+\vec{k}_{2}-\vec{k}\right) \frac{\vec{k} \cdot \vec{k}_{1}}{k_{1}^{2}} \hat{\theta}\left(\vec{k}_{1}, \tau\right) \hat{\delta}\left(\vec{k}_{2}, \tau\right)
$$




$$
\frac{\partial \hat{\theta}}{\partial \tau}+\frac{\dot{a}}{a} \hat{\theta}+\frac{6}{\tau^{2}} \hat{\delta}=-\int d^{3} k_{1} \int d^{3} k_{2} \delta_{\mathrm{D}}\left(\vec{k}_{1}+\vec{k}_{2}-\vec{k}\right) \frac{k^{2}\left(\vec{k}_{1} \cdot \vec{k}_{2}\right)}{2 k_{1}^{2} k_{2}^{2}} \hat{\theta}\left(\vec{k}_{1}, \tau\right) \hat{\theta}\left(\vec{k}_{2}, \tau\right)
$$

In equations (4) the nonlinear terms constitute the right-hand side and illustrate that the nonlinear evolution of the fields $\hat{\delta}$ and $\hat{\theta}$ at a given wavevector $\vec{k}$ is determined by the mode coupling of the fields at all pairs of wavevectors whose sum is $\vec{k}$, as required by spatial homogeneity. This makes it impossible to obtain exact solutions to the equations, so that the only general analytical technique for self-consistently evaluating the nonlinear terms is to make a perturbative expansion in $\hat{\delta}$ and $\hat{\theta}$. The formalism for such an expansion has been systematically developed by Goroff et al. (1986) and recently extended by Makino et al. (1992). Following these authors we write the solution to equations (4) as a perturbation series,

$$
\hat{\delta}(\vec{k}, \tau)=\sum_{n=1}^{\infty} a^{n}(\tau) \delta_{n}(\vec{k}), \quad \hat{\theta}(\vec{k}, \tau)=\sum_{n=1}^{\infty} \dot{a}(\tau) a^{n-1}(\tau) \theta_{n}(\vec{k})
$$

It is easy to verify that for $n=1$ the time dependent part of the solution correctly gives the linear growing modes $\delta_{1} \propto a(\tau)$ and $\theta_{1} \propto \dot{a}$ and that the time-dependence is consistent with equations (4) for all $n$. To obtain formal solutions for the $\vec{k}$ dependence at all orders we proceed as follows.

Substituting equation (5) into equations (4) yields, for $n>1$,

$$
n \delta_{n}(\vec{k})+\theta_{n}(\vec{k})=A_{n}(\vec{k}), \quad 3 \delta_{n}(\vec{k})+(1+2 n) \theta_{n}(\vec{k})=B_{n}(\vec{k}),
$$

where

$$
\begin{gathered}
A_{n}(\vec{k}) \equiv-\int d^{3} k_{1} \int d^{3} k_{2} \delta_{\mathrm{D}}\left(\vec{k}_{1}+\vec{k}_{2}-\vec{k}\right) \frac{\vec{k} \cdot \vec{k}_{1}}{k_{1}^{2}} \sum_{m=1}^{n-1} \theta_{m}\left(\vec{k}_{1}\right) \delta_{n-m}\left(\vec{k}_{2}\right) \\
B_{n}(\vec{k}) \equiv-\int d^{3} k_{1} \int d^{3} k_{2} \delta_{\mathrm{D}}\left(\vec{k}_{1}+\vec{k}_{2}-\vec{k}\right) \frac{k^{2}\left(\vec{k}_{1} \cdot \vec{k}_{2}\right)}{k_{1}^{2} k_{2}^{2}} \sum_{m=1}^{n-1} \theta_{m}\left(\vec{k}_{1}\right) \theta_{n-m}\left(\vec{k}_{2}\right)
\end{gathered}
$$

Solving equations $(6)$ for $\delta_{n}$ and $\theta_{n}$ gives, for $n>1$,

$$
\delta_{n}(\vec{k})=\frac{(1+2 n) A_{n}(\vec{k})-B_{n}(\vec{k})}{(2 n+3)(n-1)}, \quad \theta_{n}(\vec{k})=\frac{-3 A_{n}(\vec{k})+n B_{n}(\vec{k})}{(2 n+3)(n-1)}
$$

Equations (7) and (8) give recursion relations for $\delta_{n}(\vec{k})$ and $\theta_{n}(\vec{k})$, with starting values $\delta_{1}(\vec{k})$ and $\theta_{1}=-\delta_{1}$. The general solution may be written

$$
\begin{aligned}
& \delta_{n}(\vec{k})=\int d^{3} q_{1} \cdots \int d^{3} q_{n} \delta_{\mathrm{D}}\left(\vec{q}_{1}+\cdots+\vec{q}_{n}-\vec{k}\right) F_{n}\left(\vec{q}_{1}, \ldots, \vec{q}_{n}\right) \delta_{1}\left(\vec{q}_{1}\right) \cdots \delta_{1}\left(\vec{q}_{n}\right), \\
& \theta_{n}(\vec{k})=-\int d^{3} q_{1} \cdots \int d^{3} q_{n} \delta_{\mathrm{D}}\left(\vec{q}_{1}+\cdots+\vec{q}_{n}-\vec{k}\right) G_{n}\left(\vec{q}_{1}, \ldots, \vec{q}_{n}\right) \delta_{1}\left(\vec{q}_{1}\right) \cdots \delta_{1}\left(\vec{q}_{n}\right) .
\end{aligned}
$$


From equations (7)-(9) we obtain recursion relations for $F_{n}$ and $G_{n}$ :

$$
\begin{aligned}
F_{n}\left(\vec{q}_{1}, \ldots, \vec{q}_{n}\right)= & \sum_{m=1}^{n-1} \frac{G_{m}\left(\vec{q}_{1}, \ldots, \vec{q}_{m}\right)}{(2 n+3)(n-1)}\left[(1+2 n) \frac{\vec{k} \cdot \vec{k}_{1}}{k_{1}^{2}} F_{n-m}\left(\vec{q}_{m+1}, \ldots, \vec{q}_{n}\right)\right. \\
& \left.+\frac{k^{2}\left(\vec{k}_{1} \cdot \vec{k}_{2}\right)}{k_{1}^{2} k_{2}^{2}} G_{n-m}\left(\vec{q}_{m+1}, \ldots, \vec{q}_{n}\right)\right] \\
G_{n}\left(\vec{q}_{1}, \ldots, \vec{q}_{n}\right)= & \sum_{m=1}^{n-1} \frac{G_{m}\left(\vec{q}_{1}, \ldots, \vec{q}_{m}\right)}{(2 n+3)(n-1)}\left[3 \frac{\vec{k} \cdot \vec{k}_{1}}{k_{1}^{2}} F_{n-m}\left(\vec{q}_{m+1}, \ldots, \vec{q}_{n}\right)\right. \\
& \left.+n \frac{k^{2}\left(\vec{k}_{1} \cdot \vec{k}_{2}\right)}{k_{1}^{2} k_{2}^{2}} G_{n-m}\left(\vec{q}_{m+1}, \ldots, \vec{q}_{n}\right)\right]
\end{aligned}
$$

where $\vec{k}_{1} \equiv \vec{q}_{1}+\cdots+\vec{q}_{m}, \vec{k}_{2} \equiv \vec{q}_{m+1}+\cdots+\vec{q}_{n}, \vec{k} \equiv \vec{k}_{1}+\vec{k}_{2}$ and $F_{1}=G_{1}=1$. Equations (10) are equivalent to equations (6) and (A1) of Goroff et al. (1986), with $F_{n}=P_{n}$ and $G_{n}=(3 / 2) Q_{n}$ in their notation.

\subsection{Power Spectrum at Second Order}

To calculate the power spectrum we shall prefer to use symmetrized forms of $F_{n}$ and $G_{n}$, denoted $F_{n}^{(a)}$ and $G_{n}^{(a)}$ and obtained by summing the $n$ ! permutations of $F_{n}$ and $G_{n}$ over their $n$ arguments and dividing by $n$ !. Since the arguments are dummy variables of integration the symmetrized functions can be used in equations (9) without changing the result. The symmetrized second-order solutions of equations (10) are given by

$$
\begin{aligned}
& F_{2}^{(\lrcorner)}\left(\vec{k}_{1}, \vec{k}_{2}\right)=\frac{5}{7}+\frac{2}{7} \frac{\left(\vec{k}_{1} \cdot \vec{k}_{2}\right)^{2}}{k_{1}^{2} k_{2}^{2}}+\frac{\left(\vec{k}_{1} \cdot \vec{k}_{2}\right)}{2}\left(\frac{1}{k_{1}^{2}}+\frac{1}{k_{2}^{2}}\right), \\
& G_{2}^{(\lrcorner)}\left(\vec{k}_{1}, \vec{k}_{2}\right)=\frac{3}{7}+\frac{4}{7} \frac{\left(\vec{k}_{1} \cdot \vec{k}_{2}\right)^{2}}{k_{1}^{2} k_{2}^{2}}+\frac{\left(\vec{k}_{1} \cdot \vec{k}_{2}\right)}{2}\left(\frac{1}{k_{1}^{2}}+\frac{1}{k_{2}^{2}}\right) .
\end{aligned}
$$

Note that $F_{2}^{(\bullet)}$ and $G_{2}^{(s)}$ have first-order poles as $k_{1} \rightarrow 0$ or $k_{2} \rightarrow 0$ for fixed $\vec{k}$ : $F_{2}^{(\bullet)} \sim G_{2}^{(\bullet)} \sim(1 / 2) \cos \vartheta\left(k_{1} / k_{2}+k_{2} / k_{1}\right)$ where $\vartheta$ is the angle between $\vec{k}_{1}$ and $\vec{k}_{2}$. The expression for $F_{3}^{(\bullet)}$ will also be required, but since it is very long we shall wait to write a simplified form below.

The recursion relations in equations (10) may be used to compute the power spectrum at any order in perturbation theory. Substituting equation (5) into equation (3), we have

$$
P(k, \tau) \delta_{\mathrm{D}}\left(\vec{k}+\vec{k}^{\prime}\right)=\left\langle\delta(\vec{k}, \tau) \delta\left(\vec{k}^{\prime}, \tau\right)\right\rangle
$$




$$
\begin{aligned}
= & a^{2}(\tau)\left\langle\delta_{1}(\vec{k}) \delta_{1}\left(\vec{k}^{\prime}\right)\right\rangle+a^{4}(\tau)\left[\left\langle\delta_{1}(\vec{k}) \delta_{3}\left(\vec{k}^{\prime}\right)\right\rangle+\left\langle\delta_{2}(\vec{k}) \delta_{2}\left(\vec{k}^{\prime}\right)\right\rangle\right. \\
& \left.+\left\langle\delta_{3}(\vec{k}) \delta_{1}\left(\vec{k}^{\prime}\right)\right\rangle\right]+O\left(\delta_{1}^{6}\right) .
\end{aligned}
$$

Equation (12) explicitly shows all the terms contributing to the power spectrum at fourth order in the initial density field $\delta_{1}$ (or second order in the initial spectrum), as the $n$th order field $\delta_{n}(\vec{k})$ involves $n$ powers of $\delta_{1}(\vec{k})$. With the definition

$$
\left\langle\delta_{m}(\vec{k}) \delta_{n-m}\left(\vec{k}^{\prime}\right)\right\rangle \equiv P_{m, n-m}(k) \delta_{\mathrm{D}}\left(\vec{k}+\vec{k}^{\prime}\right)
$$

the power spectrum up to second order (i.e., fourth order in $\delta_{1}$ ) is given by equation (12) as

$$
\begin{aligned}
P(k, \tau) & =a^{2}(\tau) P_{11}(k)+a^{4}(\tau)\left[P_{22}(k)+2 P_{13}(k)\right] \\
& =a^{2}(\tau) P_{11}(k)+a^{4}(\tau) P_{2}(k),
\end{aligned}
$$

where the net second order contribution $P_{2}(k)$ is defined as

$$
P_{2}(k)=P_{22}(k)+2 P_{13}(k) \text {. }
$$

To determine $P_{2}(k)$ we need to evaluate the 4-point correlations of the linear density field $\delta_{1}(\vec{k})$. For a gaussian random field, all cumulants (irreducible correlation functions) of $\delta_{1}(\vec{k})$ vanish aside from the 2-point cumulant, which is given by equation (3) for $m=n-m=1$. All odd moments of $\delta_{1}(\vec{k})$ vanish. Even moments are given by symmetrized products of the 2-point cumulants. Thus the 4-point correlation function of $\delta_{1}(\vec{k})$ is

$$
\begin{aligned}
& \left\langle\delta_{1}\left(\vec{k}_{1}\right) \delta_{1}\left(\vec{k}_{2}\right) \delta_{1}\left(\vec{k}_{3}\right) \delta_{1}\left(\vec{k}_{4}\right)\right\rangle=P\left(k_{1}\right) P\left(k_{3}\right) \delta_{\mathrm{D}}\left(\vec{k}_{1}+\vec{k}_{2}\right) \delta_{\mathrm{D}}\left(\vec{k}_{3}+\vec{k}_{4}\right) \\
& +P\left(k_{1}\right) P\left(k_{2}\right) \delta_{\mathrm{D}}\left(\vec{k}_{1}+\vec{k}_{3}\right) \delta_{\mathrm{D}}\left(\vec{k}_{2}+\vec{k}_{4}\right)+P\left(k_{1}\right) P\left(k_{2}\right) \delta_{\mathrm{D}}\left(\vec{k}_{1}+\vec{k}_{4}\right) \delta_{\mathrm{D}}\left(\vec{k}_{2}+\vec{k}_{3}\right) .
\end{aligned}
$$

With the results and techniques described above, we can proceed to obtain the second order contribution to the power spectrum. The two terms contributing at second order simplify to the following 3 -dimensional integrals in wavevector space:

$$
P_{22}(k)=2 \int d^{3} q P_{11}(q) P_{11}(|\vec{k}-\vec{q}|)\left[F_{2}^{(0)}(\vec{q}, \vec{k}-\vec{q})\right]^{2},
$$

with $F_{2}^{(\bullet)}$ given by equation (11a), and

$$
2 P_{13}(k)=6 P_{11}(k) \int d^{3} q P_{11}(q) F_{3}^{(\triangleleft)}(\vec{q},-\vec{q}, \vec{k}) .
$$

The numbers in front of the integrals arise from the procedure of taking expectation values illustrated in equation (16). We write the integrals in spherical coordinates $q, \vartheta$, and $\phi$ : 
the magnitude, polar angle and azimuthal angle, respectively, of the wavevector $\vec{q}$. Then with the external wavevector $\vec{k}$ aligned along the $z$-axis the integral over $\phi$ is trivial and simplifies $\int d^{3} q$ to $2 \pi \int d q q^{2} \int d \cos \vartheta$. For $P_{13}$, the dependence on $\vartheta$ is also straightforward as it arises only through $F_{3}^{(s)}$ and not $P_{11}$. This allows the integral over $\cos \vartheta$ to be done analytically as well, giving (Makino et al. 1992)

$$
\begin{aligned}
2 P_{13}(k)= & \frac{2 \pi}{252} P_{11}(k) \int d q P_{11}(q)\left[12 \frac{k^{2}}{q^{2}}-158+100 \frac{q^{2}}{k^{2}}-42 \frac{q^{4}}{k^{4}}\right. \\
& \left.+\frac{3}{k^{5} q^{3}}\left(q^{2}-k^{2}\right)^{3}\left(7 q^{2}+2 k^{2}\right) \ln \left(\frac{k+q}{|k-q|}\right)\right] .
\end{aligned}
$$

Thus with a specified initial spectrum $P_{11}(k)$ equations (17) and (19) give the second order contribution. Before evaluating these integrals for the CDM initial spectrum, we point out that the poles of $F_{2}$ and $G_{2}$ described after equations (11) give the leading order part of the integrand of equation $(17)$ in $(q / k)$ as:

$$
P_{22}(k) \sim k^{2} P_{11}(k) \int \frac{d^{3} q}{3 q^{2}} P_{11}(q) \text {. }
$$

If $P_{11}(k) \sim k^{n}$ with $n \leq-1$ as $k \rightarrow 0$, then $P_{22}$ diverges. Vishniac (1983) showed that the leading order part of $2 P_{13}$ in $(q / k)$ is negative and exactly cancels that of $P_{22}$ - this can be demonstrated by examining the limiting form of $F_{3}^{(\bullet)}$. In a future paper we will analyze the leading order behavior of perturbative integrals at higher orders and also calculate it using a nonperturbative approach in order to investigate whether there may exist divergences for some power spectra at higher orders in perturbation theory. For the purposes of the second order integration the cancellation of the leading order terms has no consequence other than requiring that each piece, $P_{22}$ and $P_{13}$, be integrated very accurately to get the resultant. This is necessary because the cancelling parts cannot be removed before performing the integrals as the two integrands have different forms: $P_{22}$ is symmetric in $\vec{q}$ and $(\vec{k}-\vec{q})$, whereas $P_{13}$ is not. We will return to this point in the next section.

\section{Results for CDM}

The results obtained in the previous section will now be used to obtain the second order contributions to the CDM power spectrum. We will use the standard CDM spectrum with parameters $\Omega=1, H_{0}=50 \mathrm{~km} \mathrm{~s}^{-1} \mathrm{Mpc}^{-1}$, and $\sigma_{8}=1$. For the linear spectrum at $a=1$ we use the fitting form given by Bardeen et al. (1986):

$$
\begin{aligned}
& P_{11}(k)=A k T^{2}(k), \quad A=2.19 \times 10^{4} \mathrm{Mpc}^{4}, \\
& T(k)=\frac{\ln (1+9.36 k)}{9.36 k}\left[1+15.6 k+(64.4 k)^{2}+(21.8 k)^{3}+(26.8 k)^{4}\right]^{-1 / 4},
\end{aligned}
$$


where $k$ is in units of $\mathrm{Mpc}^{-1}$. With this initial spectrum equations (17) and (19) can be used to obtain the second order contribution $P_{2}(k)$, which can then be used to obtain the net power spectrum as a function of $a$ and $k$ from equation (14).

\subsection{Nonlinear Power Spectrum}

As pointed out in Section 2.2 the integrals for $P_{22}$ and $P_{13}$ contain large contributions which exactly cancel each other. For the CDM spectrum these contributions are finite but care is still required in their numerical evaluation. Equal contributions from $P_{22}$ are made as $\vec{q} \rightarrow 0$ and $\vec{q} \rightarrow \vec{k}$, whereas the cancelling contribution from $2 P_{13}$ is made only as $\vec{q} \rightarrow 0$. The integrand for $P_{22}$ is symmetric in $\vec{q}$ and $(\vec{k}-\vec{q})$ and is positive definite. For ease of numerical integration, we break up the integration range for $P_{22}$ as follows:

$$
\begin{aligned}
\int \frac{d^{3} q}{2 \pi}= & 2 \int_{0}^{\epsilon} d q \int_{-1}^{1} d y+\int_{\epsilon}^{k-\epsilon} d q \int_{-1}^{1} d y+\int_{k-\epsilon}^{k+\epsilon} d q \int_{-1}^{\left(k^{2}+q^{2}-e^{2}\right) / 2 k q} d y \\
& +\int_{k+\epsilon}^{k-k_{e}} d q \int_{-1}^{1} d y+\int_{k-k_{e}}^{k_{e}} d q \int_{\left(k^{2}+q^{2}-k_{e}^{2}\right) / 2 k q}^{1} d y
\end{aligned}
$$

where $y \equiv \cos \vartheta$, and $k_{c}$ is the upper limit required because at high $q$ the spectrum has departed strongly from the linear spectrum causing the perturbative expansion to break down. Transfer of power from higher frequencies is suppressed by virialization. The first term on the right-hand side of equation (22) has a factor of 2 because we have used the symmetry between $\vec{q}$ and $(\vec{k}-\vec{q})$ in the integrand to exclude a small ball of radius $\epsilon$ around $\vec{q}=\vec{k}$ (where the integration becomes difficult) by restricting the limits on $y$ in the third term, requiring us to double the contribution from a similar ball around $\vec{q}=0$ to compensate. The limits on $y$ in the last term are set to ensure that $|\vec{k}-\vec{q}| \leq k_{c}$ as required to consistently impose the upper limit, i.e., to exclude any contribution from $P_{11}$ in equation (17) when its argument exceeds $k_{c}$. It is in principle important to scale $k_{c}$ with time to reflect the growth of the nonlinear length scale with time, because that determines the range of validity of the perturbative expansion. We have done so using the linear scaling $k_{c} \propto a^{-2 /(3+\pi)}$, although as explained below at early times the result is insensitive to the choice of $k_{c}$.

The results of performing the integrals in equations (17) and (19) for a large range of values of $k$ are shown in Figure 1. We plot the linear spectrum $a^{2} P_{11}(k)$, the net spectrum including second order contributions given by equation (14), and the nonlinear spectrum computed from high-resolution N-body simulations described in Section 3.2 at four values of the expansion factor. The spectra have been divided by $a^{2}$ to facilitate comparison of the results at different times. The second order results at different values of $a$ are obtained 
by simply multiplying $P_{11}$ and $P_{2}$ by different powers of $a$ as shown in equation (14), so the integration of $P_{22}$ and $P_{13}$ needs to be done only once for a given $k$. The second order spectrum should be taken seriously only for the range of $k$ for which $a^{4} P_{2}(k)<a^{2} P_{11}(k)$, as we do not expect the perturbative results to be valid for higher $k$. The interesting range of $k$, the regime where nonlinear effects set in, moves to lower $k$ as one looks at larger $a$, reflecting the progress of nonlinearities to larger length scales (lower $k$ ) at late times. As expected we find that at a given time the second order contribution is not significant for small $k$ where the rms $\delta \rho / \rho \ll 1$.

For small $k$ up to just over the peak of the spectrum, the second order contribution is negative, causing the nonlinear spectrum to be lower than the linear one. At relatively high $k$ the second order contribution enhances the growth of the spectrum. This has the effect of making the slope of the spectrum significantly shallower at high $k$ than that of the linear spectrum. Thus, power is effectively transfered from long to short wavelengths, although the enhancement at short wavelengths exceeds the suppression at long wavelengths.

The two power law model of Makino et al. (1992) gives qualitatively similar results to those shown in Figure 1. Bond \& Couchman (1988) also computed the second order contributions to the CDM spectrum with a view to checking the reliability of the Zel'dovich approximation at the same order. They found excellent agreement, in contrast to the results of Grinstein \& Wise (1987) who found that in comparison to perturbation theory the Zel'dovich approximation significantly underestimated the magnitudes of the gaussian filtered, connected parts of the third and fourth moment of the real space density. In comparison to our results, Figure 3 of Bond \& Couchman shows a larger enhancement over the linear spectrum, and does not appear to show the suppression at relatively low $k$ at all. They do not give the explicit form of the term corresponding to our $P_{13}$, but state that it is negligible in comparison to $P_{22}$. This does not agree with our results at low $k$ and is probably the source of the difference in our figures. It is difficult to make a more detailed comparison without knowing the explicit form of their second term.

In order to obtain a better understanding of the dynamics of the mode-coupling, we have examined the relative contribution of different parts of the CDM initial spectrum to the second order results at a given $k$. Let $\vec{q}$ denote the integrated wavevector and $\vec{k}$ the external wavevector at which the second order contribution is calculated, as in equations (17) and (19). There is a two-fold ambiguity because wavevector $\vec{k}-\vec{q}$ contributes at the same time as $\vec{q}$. We have carefully examined different ways of associating second order contributions from different parts of the initial spectrum, and found that the second order contribution from $q \lesssim k$ tends to be positive and that from $q \gtrsim k$ negative. Indeed we also find this to hold for power law spectra with $-3 \leq n \leq 1$, independently of the value of $n$, 
thus indicating that it is a general feature of second-order mode-coupling. In Figure 2 we associate the second-order contributions, $d P_{2}(k) / d \ln q$, with the smaller of $q$ and $|\vec{k}-\vec{q}|$.

There are two regimes in the CDM spectrum, divided roughly by the part where the logarithmic slope $\left.n\left[\equiv d \ln P_{11}\right) / d \ln k\right]$ falls below -1 . For small $k$, where $n \gtrsim-1$, the positive second order contribution from $q \lesssim k$ is swamped by the negative contribution made by large $q$. The net effect is to decrease the growth of the spectrum compared to the linear growth. For relatively large $k$, where $n \lesssim-2$ the positive contribution from small $q$ dominates, increasingly so as one goes to higher $k$. A comparison of the curves in Figure 2 for $k=0.1 \mathrm{Mpc}^{-1}$ and $k=1 \mathrm{Mpc}^{-1}$ shows how the relative strengths of the positive and negative contributions shift as one moves across the spectrum. This shift can be understood by observing that at higher $k$ there is an increasing amount of power in the initial spectrum at $q<k$; the plot of the rms power on scale $k$ in Figure 3 illustrates this point. The increased power at small $q$ causes a larger nonlinear enhancement at higher $k$. Since the weakly nonlinear regime moves to higher $k$ at earlier times, the enhancement at high $k$ in turn leads to a stronger nonlinear growth at earlier times. We study the consequence of this fact in detail in Section 3.3. The dominance of the nonlinear contribution from long-wave modes also strengthens the consistency of the perturbative calculation, because the amplitude of the density fluctuations is small for these modes. As discussed in Section 3.2 , this may be responsible for the second order results being valid for a much larger range of scales at earlier times.

We emphasize that the transition value $n \simeq-1$ for the change in sign of the second order contribution is only approximate, because it depends on the value of $k$ taken as being representative of the weakly nonlinear regime. We have examined the second order contribution for power law spectra $P_{11}(k) \propto k^{n}$, for a range of values of $n$ between -3 and 1 to verify this transition. We find that in the weakly nonlinear regime (defined by $k \lesssim k_{\text {rl }}$, where $k_{\text {rl }}$ is the scale at which the $\operatorname{rms} \delta \rho / \rho=1$ ), the second order contribution for $n$ sufficiently larger than -1 is negative and that for $n$ sufficiently smaller than -1 is positive. For $n \simeq-1$, the contribution is negative for low $k$ and positive for high $k$ in the weakly nonlinear regime. These results are consistent with the results of Makino et al. (1992) who examined the second order contributions for $n=1,0,-1,-2$; they also found good agreement with $\mathrm{N}$-body simulations.

A possible transition in the nature of nonlinear evolution at $n=-1$ has also been explored by studying the clustering in real space in $\mathrm{N}$-body simulations by Klypin \& Melott (1992). An examination of the origin of the term providing the dominant second order enhancement suggests that the advective $(\vec{v} \cdot \vec{\nabla})$ terms in the real space fluid equations cause the change in sign of the nonlinear contribution. This interpretation is consistent 
with the fact that for $n<-1$ there is an increasing amount of power in the rms velocity field on larger scales, and this appears to cause the nonlinear enhancement of the density from long-wave modes to dominate. These arguments are by no means rigorous, and merit further exploration.

It is worth noting that for the deeply nonlinear regime the stable clustering hypothesis (Peebles 1980, Section 73) predicts that the spectrum steepens below the linear theory spectrum for $n>-2$ and rises above it for $n<-2$. Consistency with the second order results would require that at least for $-2 \lesssim n \lesssim-1$, the nonlinear spectrum first rise above the linear one in the weakly nonlinear regime and then fall below it in the deeply nonlinear regime. This is indeed seen in N-body simulations; the results of Efstathiou et al. (1988) show only hints of this feature owing to limited resolution, but it is clearly evident in simulations with higher resolution (Bertschinger \& Gelb 1991; White 1993).

The second order spectrum provides an estimate of the change in the fluctuation amplitude due to nonlinear effects in the weakly nonlinear regime. The conventional normalization is to set the rms $\delta \rho / \rho$ on a scale of $8 h^{-1} \mathrm{Mpc}$, denoted $\sigma_{8}$, equal to 1 . The rms value is computed from the power spectrum using a top-hat filter as described in Section 3.3. We find that with the linear spectrum normalized in this way, second order effects increase $\sigma_{\mathbf{8}}$ by $10 \%$. This is a smaller enhancement than found by Hoffman (1987) for the standard deviation of the density (without filtering) using the Zel'dovich approximation. The N-body spectrum shows an even smaller change in $\sigma_{8}$ than the second order spectrum, although it is difficult to estimate accurately in a box of length $50 h^{-1} \mathrm{Mpc}$.

\subsection{Comparison with N-Body Simulations}

The N-body results shown in Figure 1 are from two different particle-particle/particlemesh simulations of the CDM model in a $(100 \mathrm{Mpc})^{3}$ box normalized so that linear $\sigma_{8}=1$ at $a=1 /(1+z)=1$. For $a>0.1$ we have used the simulation with $144^{3}$ particles and Plummer softening distance $65 \mathrm{kpc}$ performed by Gelb \& Bertschinger (1993). To obtain accurate results at higher redshifts we have performed a new simulation with $288^{3}$ particles each of mass $2.9 \times 10^{9} M_{\odot}$ with Plummer softening distance $20 \mathrm{kpc}$. In both cases the energy conservation, as measured by integrating the Layzer-Irvine equation, was much better than 1 percent.

The comparison of power spectra in Figure 1 shows qualitative agreement between the second order and N-body results - in both the small dip in the spectrum at small $k$ and the enhancement at high $k$. At early times the agreement of the two nonlinear spectra is excellent. This agreement extends beyond the naive regime of validity of the second order 
results. As suggested above, the dominance of the contribution from long-wave modes to the nonlinear enhancement at early times apparently extends the regime of validity of the second order results.

At late times $(0.5 \lesssim a \lesssim 1)$ the second order results at high $k$ show a larger enhancement of the spectrum in comparison to the $\mathrm{N}$-body results. There is a significant discrepancy in the two results even within the expected regime of validity of the second order results. This discrepancy, coupled with the good agreement at early times, indicates that even in principle the second order spectrum could not have agreed with the N-Body spectrum shown in Figure 1 at all times. The simple dependence of the second order spectrum on $a$ given in equation (14) is incompatible with the dependence of the N-Body spectrum on $a$ for the full range of $k$ lying in the nonlinear regime.

A part of the discrepancy at late times could arise from the dependence of the second order results on the upper cutoff imposed on the integrals. The cutoff dependence is indeed the largest at late times: for $a \geq 0.5$ reasonable variations in $k_{c}$ can change the result typically by over $10 \%$. Another source of disagreement could be that the N-body simulations are done in a finite size box, therefore they have a small- $k$ cutoff. Since the contribution from long-wave modes is positive, excluding these modes could cause simulations to underestimate the nonlinear enhancement of power. On comparing CDM simulations in boxes of sides 100 and $640 \mathrm{Mpc}$ we do find this to be true, but the difference is very small. Thus neither of the two reasons mentioned above explain the magnitude of the disagreement between the second order and N-body spectra. A possible explanation is an inadequate suppression of the second order spectrum due to collapse on small scales, i.e., "previrialization" (Davis \& Peebles 1977; Peebles 1990). Indeed the second order contribution from $q>k$ is negative, in qualitative agreement with such a suppression, but it should not be surprising if the magnitude of the suppression is significantly underestimated. Higher order perturbative contributions may well include some of this suppression. Our analytic treatment neglects small-scale pressure and vorticity, which should also suppress the nonlinear enhancement of power. As we mention in Section 1, so far N-body studies designed to test this hypothesis have concluded that small scale effects are negligible. However these studies have not tested different initial spectra, and they have not examined the power spectrum itself with as much dynamic range as our simulations provide.

\subsection{Scaling in Time}

The nonlinear power spectrum can be used to construct statistical measures of density fluctuations in real space. These can then be used to study the most important consequence 
of the coupling of long-wave modes: a systematic change in the variation of characteristic nonlinear scales with time. We proceed to do this by first defining the rms $\delta \rho / \rho$ averaged on length scale $R$ by integrating over the power spectrum with an appropriate window function $W$ :

$$
\delta_{R}^{2}(a) \equiv\left\langle\left(\frac{\delta \rho}{\rho}\right)^{2}\right\rangle_{R}=\int d^{3} k P(a, k) W^{2}(k R) .
$$

For $W$ we shall use three different functions: a shell in $k$-space, the top-hat in real space, and the gaussian, given respectively by,

$$
\begin{gathered}
W_{\mathrm{D}}^{2}(k R)=\delta_{\mathrm{D}}(k R-1), \\
W_{\mathrm{TH}}(k R)=\frac{3[\sin (k R)-k R \cos (k R)]}{(k R)^{3}}, \\
W_{\mathrm{G}}(k R)=\exp \left[-\frac{(k R)^{2}}{2}\right] .
\end{gathered}
$$

In Figure 3 we plot $\left[4 \pi k^{3} P(a, k)\right]^{1 / 2}$, or $\delta_{R}(a)$ for $W_{\mathrm{D}}$ with $k=r^{-1}$, to illustrate what we expect for the time dependence of a characteristic nonlinear scale, denoted as $R_{\mathrm{nl}}(a)$. If the spectrum evolved self-similarly then one would expect that at all $a$, the onset of nonlinear effects occurs at a scale defined by setting $4 \pi k^{3} P(a, k)=$ constant for some value of the constant of order unity. This behavior is expected for power law spectra of the form $P(k) \propto k^{n}$, and has been verified in studies of N-body simulations (Efstathiou et al. 1988). Even though CDM-like spectra are not pure power laws, the simplest assumption would be that they show a similar behavior. However, Figure 3 shows that at early times (small $a$ ), the spectrum deviates from the linear one at progressively smaller values of $4 \pi k^{3} P(a, k)$. This trend is even stronger for the N-body spectra. Thus already there is a hint of a systematic departure of the nonlinear scaling from the conventional expectation, due to the variation of the spectral index $n$ with scale for the CDM spectrum.

This conclusion is confirmed by using the other window functions to define the nonlinear scale as follows. We calculate $\delta_{R}(a)$ using equations $(24 b, c)$ for a range of $R$ and $a$. We then define $R_{\mathrm{nl}}(a)$ as follows:

$$
\delta_{R}(a)=\delta_{c} \quad \text { for } \quad R=R_{\mathrm{nl}}(a)
$$

where $\delta_{c}$ is a constant of order unity. Since $R_{\mathrm{nl}}(a)$ is a comoving length scale, it can be used to define a nonlinear scale for the mass as: $M_{\mathrm{nl}}(a)=(4 \pi / 3) \bar{\rho} R_{\mathrm{nl}}^{3}(a)$, where $\bar{\rho}$ is the critical density today.

In Figure 4 we have plotted $M_{\mathrm{nl}}(a)$ from $a=0.04$ to 1 for the gaussian and top-hat filters, with $\delta_{c}$ chosen to be 1 and 1.69 for each filter. The dependence of $M_{\mathrm{nl}}(a)$ confirms 
the impression conveyed by Figure 3: nonlinear enhancement is stronger at earlier times. While the quantitative results depend on the choice of the window function and $\delta_{c}$, it is clear that in each of the figures the slope of the second order curve is different from the linear curve, and this causes the relative enhancement of $M_{\mathrm{nl}}(a)$ to be larger at earlier times. Indeed, if the normalization of the second order curves was changed (thus shifting them to the right) so that at $a=1$ they predicted the same nonlinear mass as the linear curves, then all four panels would show very similar relative enhancements at early times.

In stating quantitative results for the time-dependence of nonlinear masses we shall focus on the gaussian filter with $\delta_{c}=1$. This choice provides the most conservative estimates of second order effects. At $a^{-1}=(1+z)=(20,10,5,2), M_{\mathrm{nl}}(a)$ from the second order spectrum is about $(180,8,2.5,1.6)$ times (respectively) larger than the linear case. Figure 4 can also be used to read off the change in the redshift of nonlinearity for the desired mass scale due to second order effects. (Here as in the preceding figures, the linear spectrum is normalized so that $\sigma_{8}=1$ at $z=0$ and this fixes the normalization of the second-order spectrum.) For example, the mass scale $10^{6} M_{\odot}$ goes nonlinear at $(1+z) \simeq 25$ as opposed to 19 if only the linear spectrum is used; and the mass scale $10^{11} M_{\odot}$ at $(1+z) \simeq 6$ as opposed to 5 . This change in redshift is a more meaningful indicator of the nonlinear effect, as the change in $M_{\mathrm{nl}}(a)$ is amplified due to the steepening of the spectrum at high $k$.

In Figure 4 we have also shown results from the Press-Schechter model (Press \& Schechter 1974, hereafter PS). The PS model is a widely used ansatz for predicting the distribution of bound objects of a given mass at different times (section 3.4). It relies on the linear growth of the power spectrum, hence it is no surprise that the shape of the PS curve is very similar to the linear curve. Here the PS nonlinear mass is defined as the mass for which a fixed fraction, 0.4 , of the mass in nonlinear clumps belongs to clumps of mass $M_{\mathrm{nl}}$ or larger. The fraction 0.4 is chosen so that the normalization of the PS curves is close to that of the other curves at $a=1-$ in the upper panels it is close to the nonlinear curves and in the lower panels to the linear curves.

The N-body simulations can be used to define a characteristic nonlinear mass in many different ways. The dashed curves in Figure 4 show nonlinear masses computed using the power spectrum from the simulation in the same way as for the second order and linear spectra above (i.e., using eq. 25). The results are in very good agreement with the second order results, as expected because of the good agreement of the second order and nonlinear power spectra. The relative enhancement of $M_{\mathrm{nl}}(a)$ over the linear prediction at $(1+z)=(10,5,2)$ is $(11,2.4,1.2)$. By examining all four panels it can be seen that, independent of the parameters used, the slopes of the curves using second-order and nonlinear power spectra are distinctly different from those of the linear and PS curves. The 
filled triangles use a different definition of the $\mathrm{N}$-body characteristic nonlinear mass and will be discussed in the next section.

Our results in Figure 4 indicate that linear scaling for $M_{\mathrm{nl}}(a)$ significantly underestimates nonlinear enhancement at high redshift. Consequently the characteristic masses predicted by the PS model are much smaller than the second order and $\mathrm{N}$-body masses for $z>4$, even for the choice of parameters for which they agree at late times. This conclusion may appear at odds with previous tests of the PS formalism made by others. However, no previous tests have examined the CDM model at high redshift with as much dynamic range as we have. As we have emphasized, mode-coupling from long waves is strongest for small $n$; for the CDM spectrum $n$ varies with scale and approaches -3 at high $k$. It is precisely in this limit, previously untested with high-resolution $\mathrm{N}$-body simulations, that we find the greatest departures from linear theory and the Press-Schechter model.

\subsection{Distribution of Nonlinear Masses}

The characteristic nonlinear masses defined above do not fully characterize the distribution of dense clumps that form as a result of gravitational instability. A better comparison of theory and simulation can be made using the complete distribution of masses.

In the $\mathrm{N}$-body simulation we have identified dense clumps at mean overdensity about 200 using the friends-of-friends (FOF) algorithm with linking distance 0.2 times the mean interparticle separation. (The $N=288^{3}$ simulation was used at $1+z=10$, while the $N=144^{3}$ simulation was used for $1+z=5,2$, and 1.) The distribution of nonlinear clump masses is very broad, so there is no unique nonlinear mass. We have chosen to define the characteristic nonlinear mass $M_{\mathrm{nl}}$ for this distribution as the median clump mass defined so that half of the mass in clumps of at least 5 particles is contained in clumps more massive than $M_{\text {nl }}$. The 5 particle limit corresponds to $M=1.16 \times 10^{11} M_{\odot}$ and $1.45 \times 10^{10} M_{\odot}$ for the $N=144^{3}$ and $288^{3}$ simulations, respectively. The resulting nonlinear masses are denoted by the filled triangular symbols in the upper-left panel of Figure 4; for other panels these points would be at the same locations as in this panel. (If the PS curves were defined with the same lower limit for clump masses and the same value of the mass fraction, instead of having no lower limit and a mass fraction 0.4, they would agree more closely with the $\mathrm{N}$-body FOF points.) It is coincidental that this definition of $\mathrm{N}$-body nonlinear mass yields such close agreement with the analytic predictions at $z=0$, because the broad range of clump masses would allow us to vary $M_{\mathrm{nl}}$ by factors of a few. The relative variations as a function of redshift are more meaningful. It is clear from Figure 4 that the variation of these N-body masses with $a$ departs from the linear scaling even more strongly than the 
curve computed from the N-body power spectrum. Thus, nonlinear effects on the formation of high-redshift objects appear to be even more significant than they are on the power spectrum. However, the 5 particle limit affects the FOF characteristic mass (no lower limit is imposed on the PS curve), so we should make a more detailed comparison with the mass distribution before reaching firm conclusions.

The PS model makes the ansatz that the formation of bound objects is determined by the overdensity in the linear density field. Using the gaussian distribution of the linear density field, this ansatz gives the comoving number density, $n(M, a)$, of nonlinear objects of mass $M$ in the mass interval $d M$ at expansion factor $a$ as (Press \& Schechter 1974):

$$
n(M, a) d M=\bar{\rho}\left(\frac{2}{\pi}\right)^{1 / 2} \frac{\delta_{c}}{\sigma} \exp \left(-\frac{\delta_{c}^{2}}{2 \sigma^{2}}\right) \frac{1}{\sigma}\left(\frac{d \sigma}{d M}\right) \frac{d M}{M} .
$$

In this equation $\delta_{c}$ is a free parameter which can be taken to be a constant, with the linear rms density smoothed on the mass scale $M, \sigma(M, a)$, growing in proportion to $a$. A popular choice for $\delta_{c}$ is 1.69 , the value of the linear density at which a spherical top-hat perturbation collapses to infinite density. The PS mass distribution $n(M, a)$ has been tested against $\mathrm{N}$-body simulations and found by other workers to work very well. Efstathiou et al. (1988) tested it for scale-free simulations, and several authors have tested it for the CDM spectrum (e.g., Carlberg \& Couchman 1989). The weaknesses of such tests - particularly, the finite resolution of the simulations - have been recognized by these authors, but even so the agreement has been surprisingly good for the range of masses and redshift probed. Consequently, the PS model has been widely used in predicting the number density of objects at high redshift, or in estimating the redshift at which a given mass scale goes nonlinear.

Figure 5 shows the cumulative mass fraction (CMF) as a function of clump mass from the $\mathrm{N}$-body simulation and the PS prediction. The CMF is defined by

$$
\operatorname{CMF}(M, a)=\bar{\rho}^{-1} \int_{M}^{\infty} n(M, a) M d M
$$

i.e., the fraction of mass in objects of mass $M$ or larger. In Figure 4 we defined the PS nonlinear mass using the condition $\mathrm{CMF}=0.4$. For the PS prediction of Figure 5 we have chosen the top-hat filter with $\delta_{c}=1.69$. At late times, this choice gives fairly good agreement for the high-mass end of the mass distribution. However, we see that at early times the N-body mass distribution lies systematically above the PS prediction. This is in qualitative agreement with the results shown in Figure 4 and supports our conclusion that nonlinear effects on the formation of nonlinear clumps are even stronger than they are for the power spectrum. 
Because coupling of long waves modifies the power spectrum and therefore the rms density $\sigma(M, a)$, the failure of Press-Schecter theory to match the N-body results exactly does not surprise us. As an experiment we replaced $\sigma(M, a)$ in equation (26) using the second-order and N-body power spectra instead of linear theory. The resulting $\operatorname{CMF}(M)$ falls too rapidly at large $M$, even after $\delta_{c}$ is increased to compensate for the nonlinear enhancement of density fluctuations. If the nonlinear power spectrum is used the PS formula gives the wrong shape for $n(M, a)$ because it assumes a gaussian distribution of densities, while the nonlinear density field has a broader distribution. We have found no simple modification of the PS formula that can account for the systematic departures evident in Figure 5. Expressing an optimistic view, we note that the PS formula is accurate to about a factor of 2 for the CMF over the entire range shown in Figure 5. On the other hand, the deviations are larger for rarer objects (smaller CMF) and the sign and magnitude of the deviation changes systematically with $a$. Therefore one should use the PS formula, especially at high redshift and for rare objects, only with caution after calibration by high-resolution $\mathrm{N}$-body simulations.

\section{Discussion}

We have calculated the second order contribution to the evolution of the standard CDM power spectrum. We believe that our results capture the dominant nonlinear contribution in the weakly nonlinear regime. They are consistent with $\mathrm{N}$-body results in this regime from $z=9$ to $z \simeq 1$, but show a larger enhancement of the spectrum than the N-body results from $z \simeq 1$ to $z=0$. The bulk of the second order enhancement in the growth of the power spectrum is provided by the mode coupling of long-wave modes, especially for the onset of nonlinearities at high redshift.

By analyzing the perturbative integrals we have studied the sensitivity of nonlinear evolution to different parts of the spectrum, and thus have probed the dynamics of the mode-coupling at work. We find that on scales of interest to large-scale structure in the universe, the dominant contribution to the weakly nonlinear evolution of most realistic power spectra comes from the mode-coupling of long-wave modes. Perturbation theory is quite adequate for estimating this contribution since the amplitude of density fluctuations is small for the long-wave modes.

An important consequence of nonlinear evolution is to change the time dependence of the nonlinear scale $M_{\mathfrak{n l}}(a)$ from linear scaling: it is found to be significantly larger at high $z$. Thus objects of a given scale go nonlinear at higher redshifts than indicated by the standard linear extrapolation. As discussed in Section 3, this is a consequence of the variation with 
scale of the spectral index, with $n \gtrsim-1$ on the scales of interest for large-scale structure and $n \simeq-3$ on the smallest scales. We have given quantitative estimates of this effect for the standard CDM spectrum for different window functions and definitions of nonlinear scale. For a gaussian window function and $\delta_{c}=1$, which provides the most conservative estimates, the change in the redshift factor of nonlinearity, $\left(1+z_{n l}\right)$, is about $20 \%$ for $10^{11} M_{\odot}$ objects (with linear extrapolation $1+z_{n l}=5$ ) and increases to about $33 \%$ for $10^{6} M_{\odot}$ objects $\left(1+z_{n l}=19\right)$. We have also computed nonlinear corrections using high resolution $\mathrm{N}$-body simulations, using the power spectrum from the simulations as well as directly identifying bound objects. The results are in very good agreement with the second order predictions, especially between $z \simeq 4$ and 10. Quantitative comparisons are provided in Sections 3.3 and 3.4 and in Figure 4.

Thus the most striking implications of second order effects are for the formation of nonlinear objects at high $z$. Theoretical studies of, for example, the first generation of collapsed objects, the redshift of galaxy-formation, and reionization at high- $z$ (see e.g., Couchman \& Rees 1986; Efstathiou \& Rees 1988; Tegmark \& Silk 1993) - all require as an input the scale of nonlinearity as a function of $z$. For analytical estimates this is invariably obtained using linear extrapolation, as for example in the Press-Schechter mass distribution. We have shown (Figure 5) that the Press-Schechter theory leads to a systematic underestimate of the abundance of high-mass nonlinear clumps at high redshift in the CDM model, but have not succeeded in suggesting a simple modification that works better. While nonlinear coupling to long waves increases the amplitude of small-scale density fluctuations, it also changes the probability distribution from the gaussian distribution appropriate in the linear regime.

Most realistic cosmological spectra steepen to $n \simeq-3$ at the smallest scales and have $n \gtrsim-1$ on the largest scales of interest. This is a generic feature arising from the sluggish, logarithmic growth of fluctuations during the radiation dominated era, thus causing the scale invariant spectrum with spectral index $n=1$ initially to approach $n=-3$ on the smallest scales while retaining the primeval slope on scales much larger than the size of the horizon at the end of the radiation dominated era. Hence for different cosmological models the basic features of nonlinear gravitational evolution that we have studied should hold, although the quantitative details would depend on the values of parameters such as $\sigma_{8}, \delta_{c}$, $\Omega$ and $H_{0}$.

The increase in redshift of collapse relative to linear theory that we have calculated for CDM should also occur in all realistic spectra provided that on the scales of interest $n$ decreases sufficiently rapidly with increasing $k$. Our results will not apply if the dark matter is hot, but the qualitative implications should be the same for the evolution of 
the baryonic component in a CDM- or baryon-dominated model until dissipational effects become important. For spectra with a very steep slope at small scales (such as in the hot dark matter model), second order effects may lead to a strong nonlinear enhancement which would drive the spectrum to a shallower slope.

In the near future second order power spectra from theoretical models could be related to the power spectrum calculated from observational surveys. Indeed the shape of the best fit three-dimensional power spectrum computed from results of the APM survey (Baugh \& Efstathiou 1993) shows two characteristic features of the second order CDM spectrum: a relatively shallow slope at small scales and a flattening of the peak of the spectrum at large scales. The power spectrum computed from the CfA redshift survey (Vogeley et al. 1992) and from the 1.2Jy IRAS redshift survey (Fisher et al. 1993) had also shown the first feature of a shallow slope with $n$ just below -1 at high $k$, but these surveys lacked the depth required to determine the shape of the spectrum near the peak. It will be interesting to see if the extended peak of the APM spectrum is a robust feature.

It is a pleasure to thank Alan Guth for many stimulating discussions. We also acknowledge useful discussions with Carlton Baugh, George Efstathiou, Yehuda Hoffman, Roman Juszkiewicz, David Weinberg and Simon White. We thank John Bahcall for his hospitality at the Institute for Advanced Study, where this work was completed. Supercomputing time was provided by the Cornell National Supercomputer Facility and the National Center for Supercomputing Applications. This work was supported by NSF grant AST90-01762. 


\section{REFERENCES}

Bardeen, J.M., Bond, J.R., Kaiser, N., \& Szalay, A.S. 1986, ApJ, 304, 15

Baugh, C. \& Efstathiou, G. 1993, MNRAS, 265, 145

Beacom, J. F., Dominik, K. G., Melott, A. L., Perkins S. P. \& Shandarin, S. F. 1991, ApJ, 372,351

Bertschinger, E. 1992, in New Insights into the Universe, ed. Martinez, V. J., Portilla, M. \& Saez, D. (Berlin: Springer-Verlag), p. 65

Bertschinger, E. \& Gelb, J. 1991, Comp. in Physics, 5, 164

Bertschinger, E. \& Jain, B. 1993, submitted to ApJ

Bond, J.R. \& Couchman, H.M.P. 1988, in Proceedings of the Second Canadian Conference on General Relativity and Relativistic Astrophysics, ed. Coley, A.A. \& Dyer, C. (Singapore: World Scientific), p. 385

Coles, P. 1990, MNRAS, 243, 171

Carlberg, R.G. \& Couchman, H.M.P. 1989, ApJ, 340, 47

Couchman, H. M. P. \& Rees, M. J. 1986, MNRAS, 221, 53

Davis, M. \& Peebles, P. J. E. 1977, ApJS, 34, 425

Efstathiou, G. 1990, in Physics of the Early Universe, , ed. Peacock, J. A., Heavens, A. F. \& Davies, A. T. (Bristol: IOP), p. 361

Efstathiou, G. \& Rees, M. J. 1988, MNRAS, 230, 5P

Efstathiou, G., Frenk, C. S., White, S. D. M., \& Davis, M. 1988, MNRAS, 235, 715

Evrard, A. E., \& Crone, M. M. 1992, ApJ, 394, L1

Fisher, K. B., Davis, M., Strauss, M. A., Yahil, A., \& Huchra, J. P. 1993, ApJ, 402, 42

Fry, J. N. 1984, ApJ, 279, 499

Gelb, J. M., \& Bertschinger, E. 1993, submitted to ApJ

Goroff, M. H., Grinstein, B., Rey, S.-J. \& Wise, M. B. 1986, ApJ, 311, 6 
Grinstein, B. \& Wise, M.B. 1987, ApJ, 320, 448

Gramann, M. 1992, ApJ, 401, 19

Gunn, J. E. \& Gott, J. R. 1972, ApJ, 176, 1

Hoffman, Y. 1987, ApJ, 318, L7

Juszkiewicz, R. 1981, MNRAS, 197, 931

Juszkiewicz, R., Sonoda, R. H. \& Barrow, J. D. 1984, MNRAS, 209, 139

Klypin, A. A. \& Melott, A. A. 1992, ApJ, 399, 397

Little, B., Weinberg, D. H. \& Park, C. 1991, MNRAS, 253, 307

Makino, N., Sasaki, M. \& Suto, Y. 1992, Phys.Rev.D, 46, 585

Peebles, P. J. E. 1980, The Large-Scale Structure of the Universe, (Princeton: Princeton University Press)

Peebles, P. J. E. 1990, ApJ, 365, 207

Press, W. H. \& Schechter, P. 1974, ApJ, 187, 425

Ryden, B. S. \& Gramann, M. 1991, ApJ, 383, L33

Shandarin, S. F. \& Melott, A. L. 1990, ApJ, 364, 396

Suto, Y. \& Sasaki, M. 1991, Phys. Rev. Lett., 66, 264

Tegmark, M. \& Silk, J. 1993, preprint

Vishniac, E. T. 1983, MNRAS, 203, 345

Vogeley, M. S., Park, C., Geller, M. J. \& Huchra, J. P. 1992, ApJ, 391, L5

White, S. D. M. 1993, private communication

Zaroubi, S. \& Hoffman, Y. 1993, ApJ, 414, 20

Zel'dovich, Ya. B. 1965, Adv. Astron. Ap., 3, 352 


\section{Figure Captions}

Fig. 1: Linear and nonlinear power spectra at expansion factors $a=0.1,0.2,0.5$, and 1 , where $a=1$ corresponds to linear $\sigma_{8}=1$. The linear spectrum is given by the dotted curves, the corresponding second order spectrum $\left[P(k)=a^{2} P_{11}(k)+a^{4} P_{2}(k)\right]$ by the solid curves and the spectrum from high resolution $\mathrm{N}$-body simulations by the dashed curve. The spectra are all divided by $a^{2}$ to facilitate better comparison of the nonlinear effects at different values of $a$. The triangles marked on the second order spectra indicate the point at which $a^{4} P_{2}(k)=a^{2} P_{11}(k)$ : this indicates the approximate limit of validity of the second order results.

Fig. 2: Contributions to $P_{2}(k)$ vs. $\log q$, where $q$ is the magnitude of the integrated wavevector. The two panels are for different choices of $k . P_{2}(k)$ is defined in equation (15) and is the sum of the contributions $P_{22}(k)$ and $2 P_{13}(k)$. The integrand of $P_{22}(k)$ is symmetric in $\vec{q}$ and $(\vec{k}-\vec{q})$; we have chosen to associate the contribution from such a pair of wavevectors with the wavevector with smaller magnitude. Other choices do not alter the basic trend seen here, namely, that the contribution from $q<k$ is generally positive and peaked at $q=k / 2$, while that from $q>k$ is generally negative. Moreover, a comparison of the plots for the two values of $k$ shown illustrates that at higher $k$ the positive contribution from small $q$ dominates, leading to a net enhancement of small-scale power. This is due to the increasing amount of power at $q<k$ for higher $k$, as can be seen in Figure 3.

Fig. 3: RMS amplitude of density fluctuations vs. scale $k$ for several expansion factors. The lower curves correspond to smaller $a$. Solid (dashed) curves are used for the second order (linear) results. The second order curves are shown only for the estimated regime of validity shown in Figure 1. It is clear from the results at different $a$ that the nonlinear contribution becomes significant at earlier $a$ for successively smaller values of $\left[4 \pi k^{3} P(a, k)\right]^{1 / 2}$.

Fig. 4: Growth of characteristic nonlinear mass with time. The mass scale $M_{\mathrm{nl}}(a)$ at which the rms $\delta \rho / \rho$ reaches a fixed value (denoted by $\delta_{c}$ in the figures) is plotted vs. the expansion factor $a=1 /(1+z)$. Two values of $\delta_{c}$ are used to define $M_{\mathrm{nl}}(a): \delta_{c}=1$ on the left and $\delta_{c}=1.69$ on the right. For each $\delta_{c}$ the rms $\delta \rho / \rho$ is computed with a gaussian window function for the upper panels, and with a real space top-hat for the lower panels. The dotted curves show $M_{\mathrm{nl}}(a)$ computed using the linear spectrum $P_{11}(k)$; the solid curves include the second order contribution for the same normalization of the linear spectrum. The dot-dashed curves have been computed from the $\mathrm{N}$-body power spectrum shown in Figure 1. The dashed curves are computed using the Press-Schechter model, with the characteristic nonlinear mass defined as that at which a fixed fraction, 0.4 , of the mass in nonlinear clumps is in clumps more massive than $M_{\mathrm{nl}}$. In the top-left panel the 
symbols labelled "N-body FOF" are obtained from the N-body simulation by using the friends-of-friends algorithm to identify clumps of at least 5 particles, and then to define a characteristic mass so that half the mass in clumps is in clumps more massive than $M_{\mathrm{nl}}$.

Fig. 5: Cumulative mass fraction (CMF) vs. clump mass $M$ at $a=0.1,0.2,0.5,1$. The dashed curves represent the predictions of the Press-Schechter model, while the solid curves are obtained from $\mathrm{N}$-body simulations. The curves are shown at different times, with the higher curves representing larger values of $a$. The $\mathrm{N}$-body curves are obtained using the friends-of-friends algorithm with linking parameter $=0.2$. 


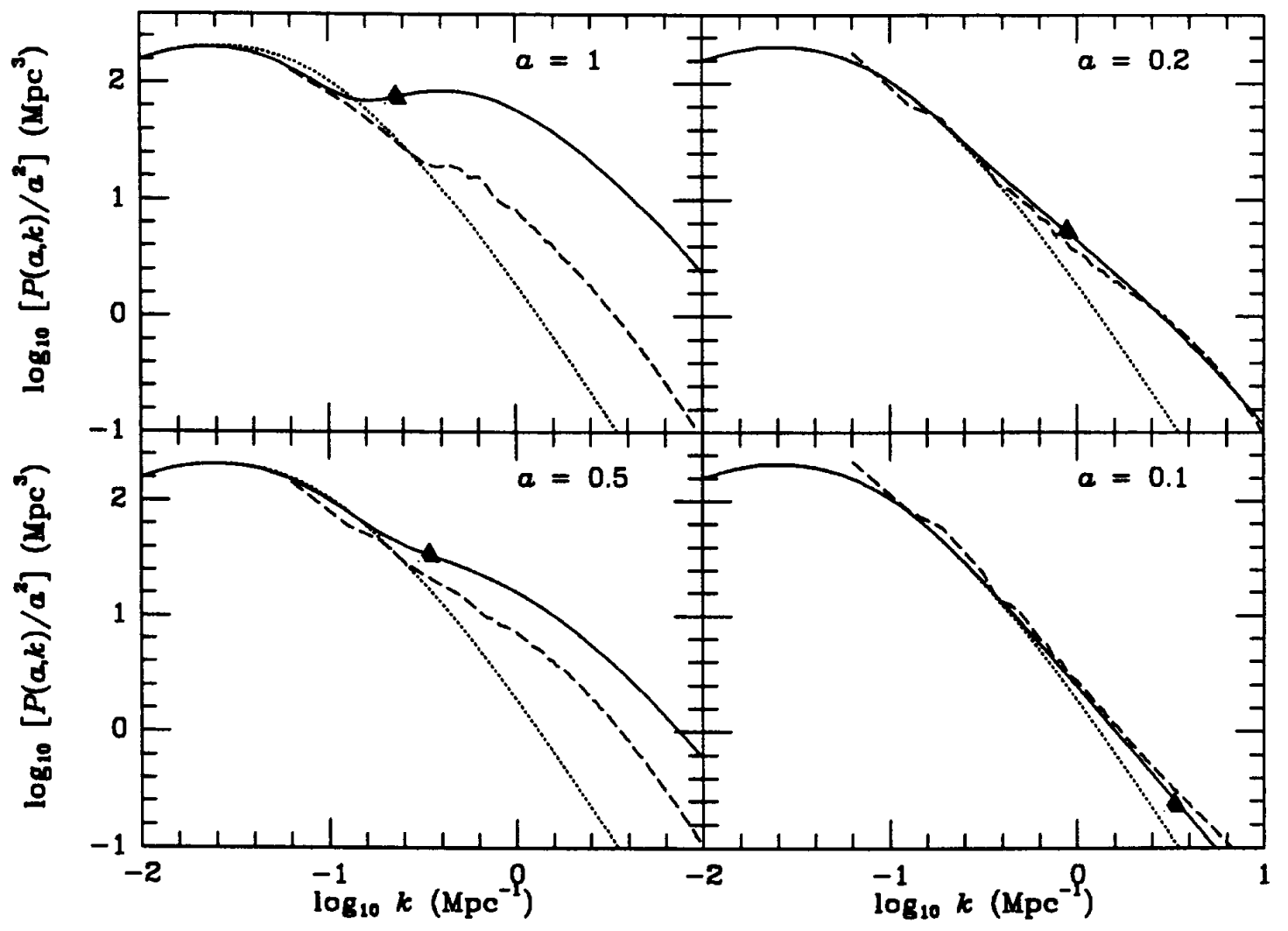

Figure 1 

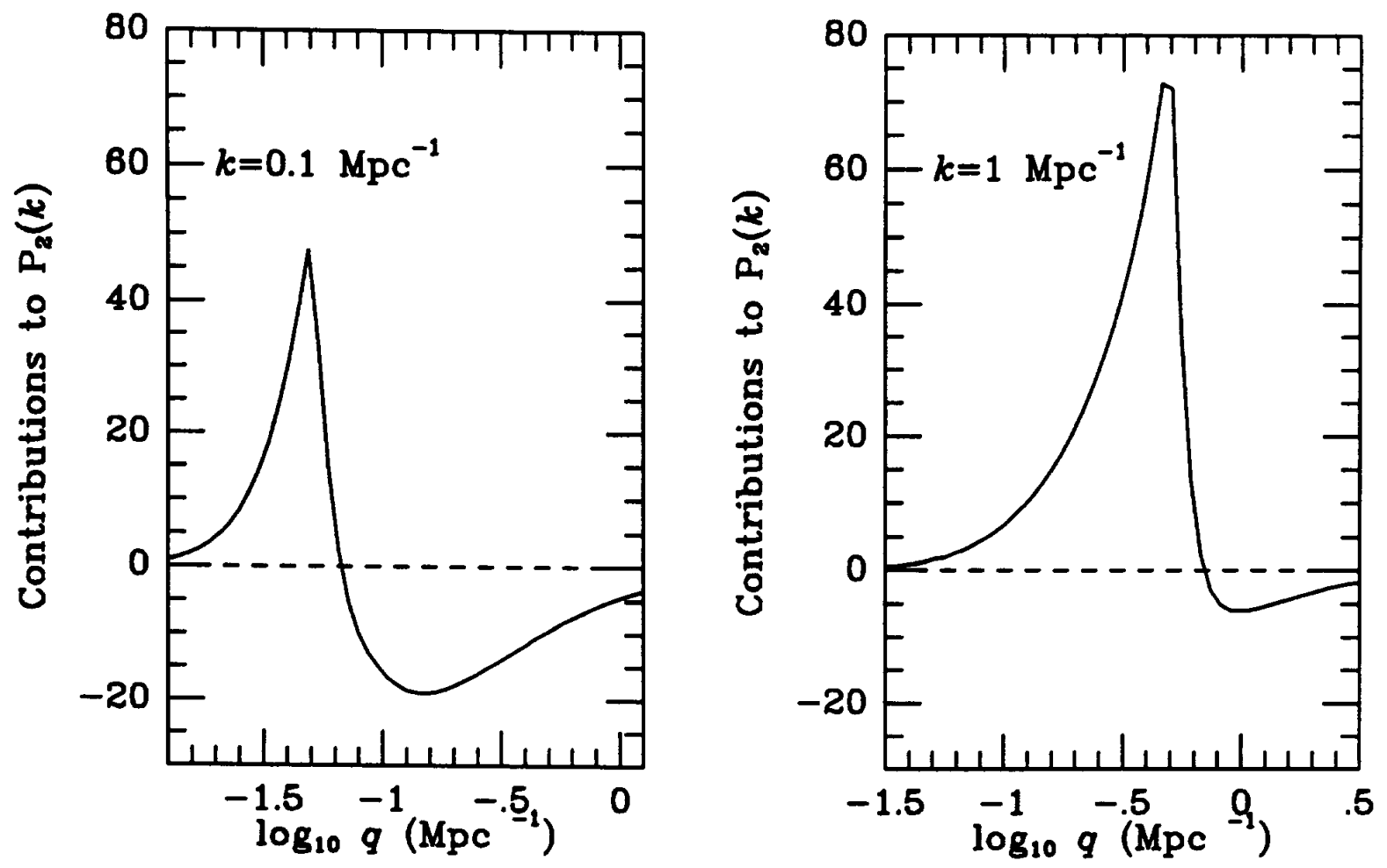

Figure 2 


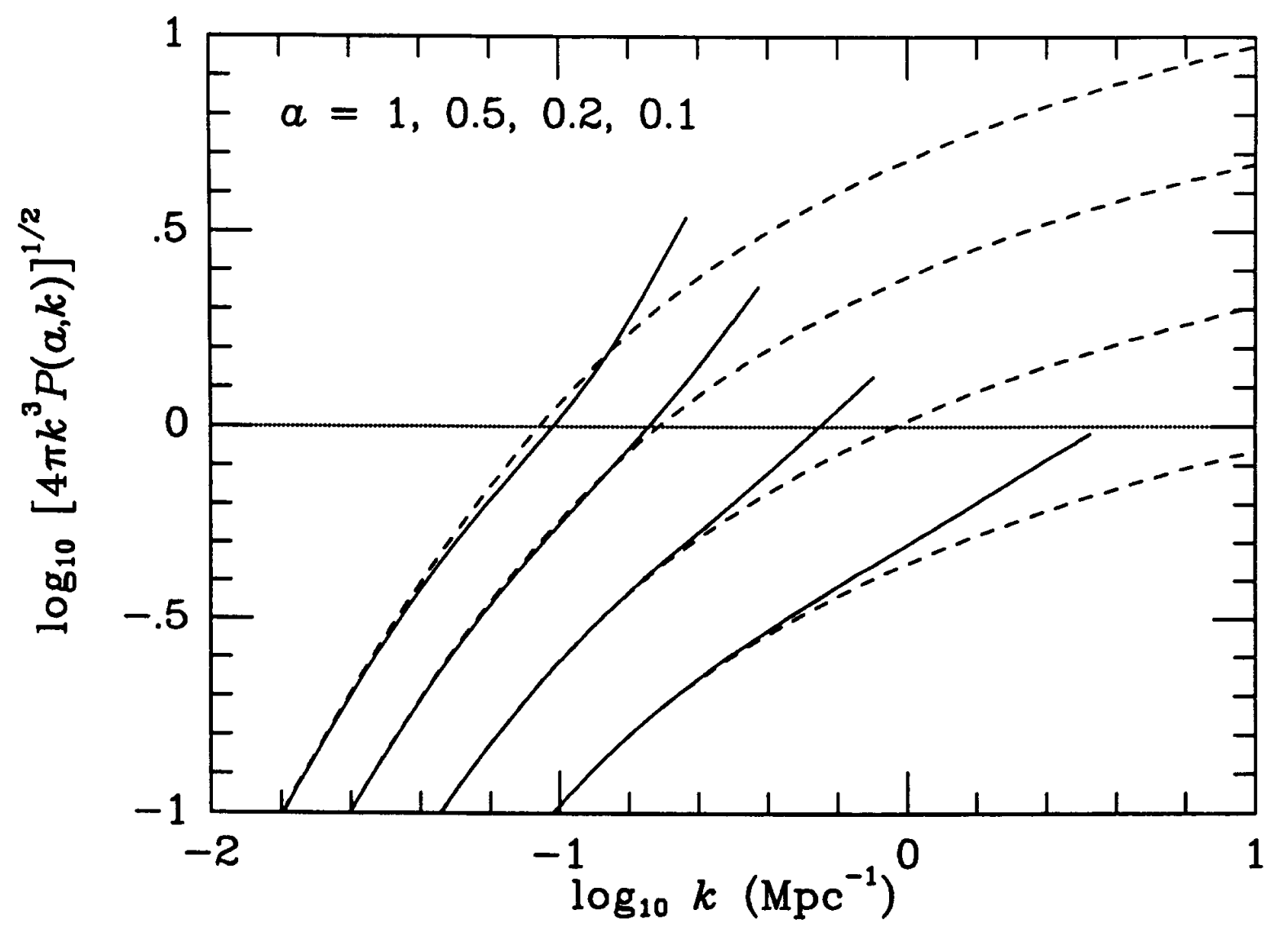

Figure 3 


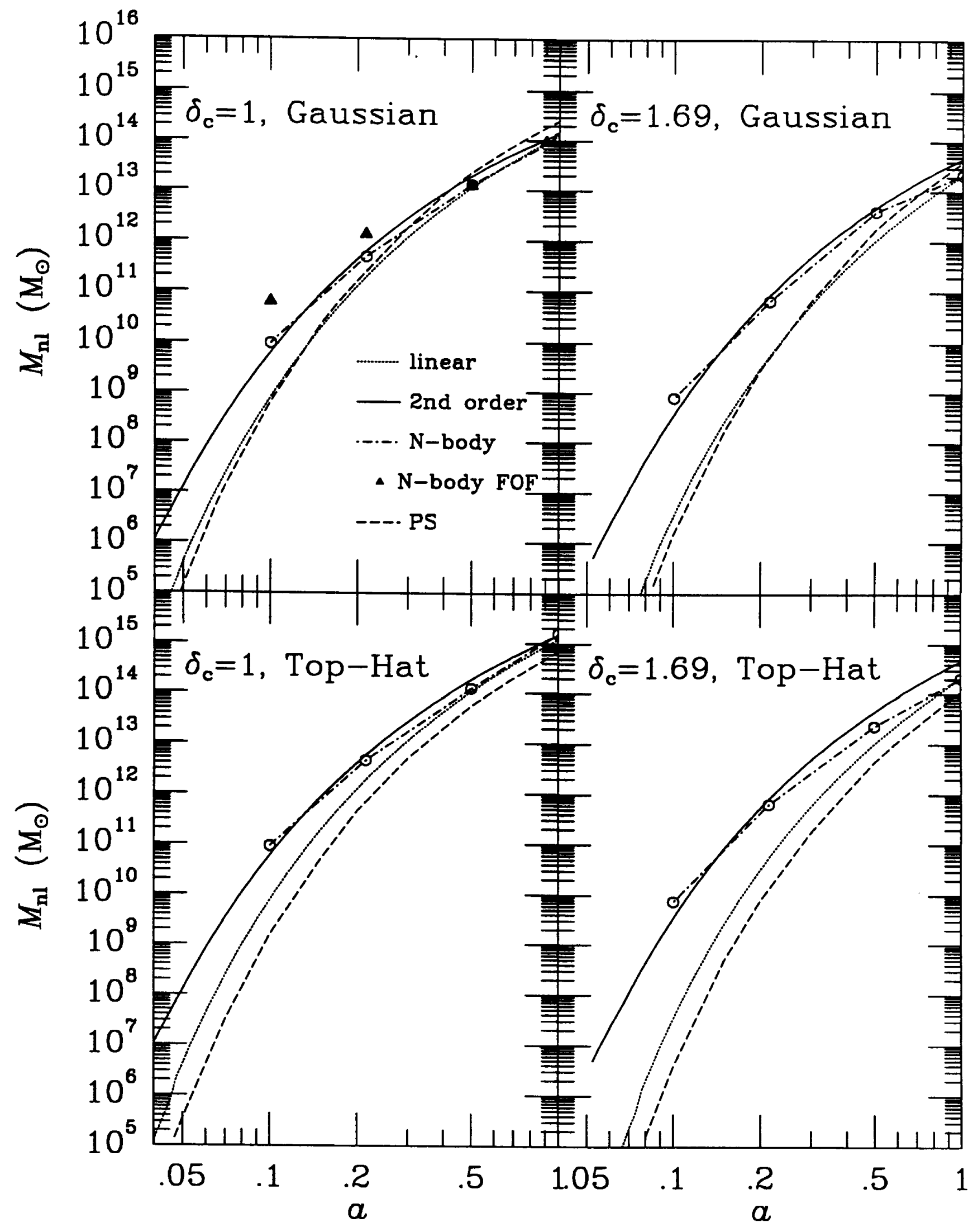

Figure 4 


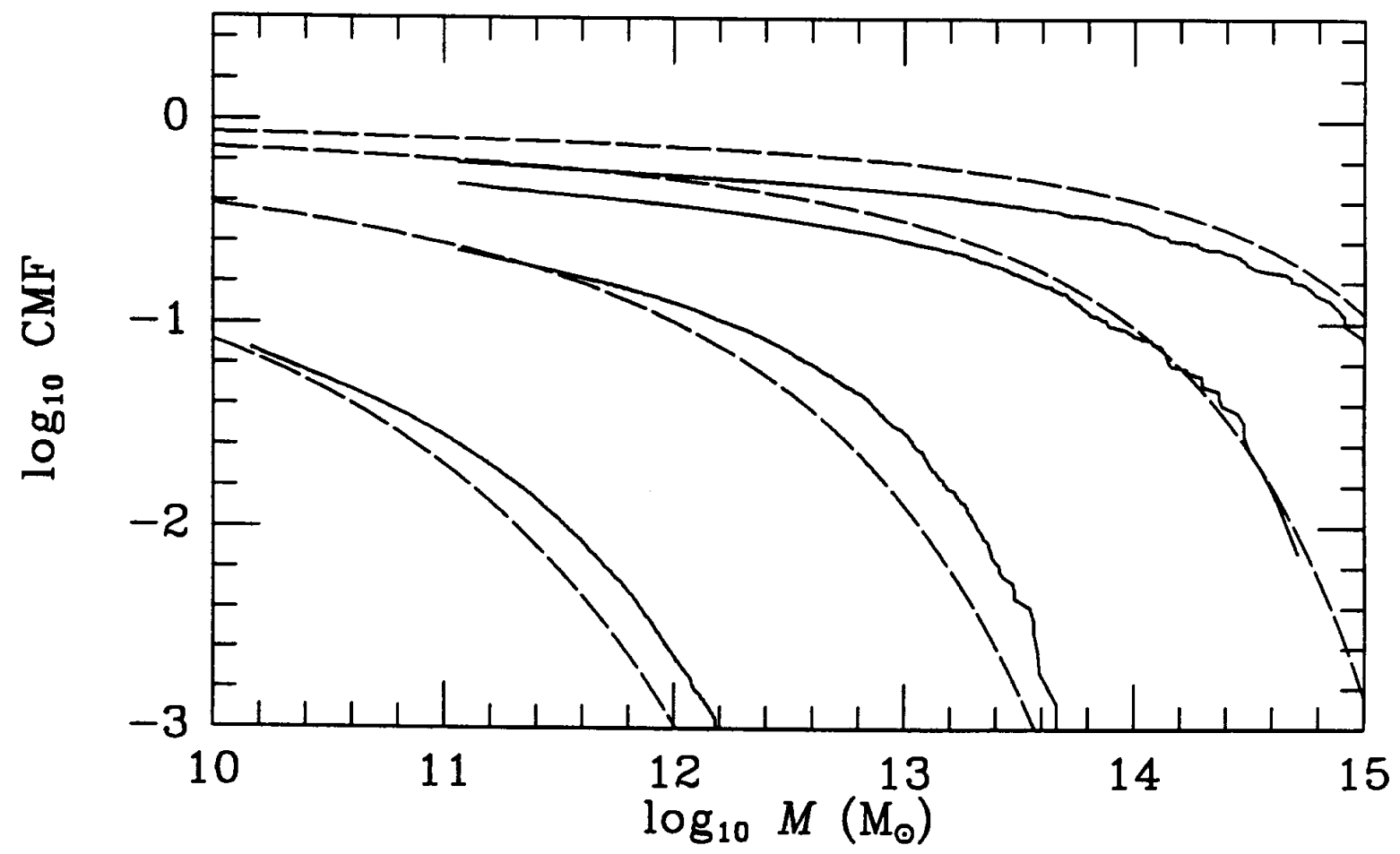

Figure 5 